\title{
Tuméfaction mandibulaire révélatrice d'un myélome multiple : à propos d'un cas
}

\section{Mandibular tumefaction revealing multiple myeloma: a case report}

\author{
CHIRAZ HAFSA ${ }^{1}$, TOUHAMI BEN ALAYA ${ }^{1,2}$, SOULEF KRIAA ${ }^{1}$, HAYKEL DEBBABI ${ }^{1}$, MOHAMED BEN KHELIFA ${ }^{3}$, \\ MONDHER GOLLI', HABIB HAMDI ${ }^{3}$, AMOR GANNOUNI ${ }^{1}$
}

\section{RÉSUMÉ}

Les auteurs rapportent le cas d'une tuméfaction mandibulaire droite survenant chez un homme âgé de 73 ans qui a amené à la découverte d'un myélome multiple. La radiographie standard de la mandibule a révélé l'existence d'une lyse osseuse de la branche montante droite de la mandibule avec une masse envahissant les parties molles. L'examen tomodensitométrique avait montré à coté du processus tumoral de la branche montante de la mandibule, des images lacunaires de la voûte du crâne et une lyse de la $3^{e}$ vertèbre cervicale faisant suspecter le diagnostic de myélome multiple. Le diagnostic positif a été confirmé par le bilan biologique et radiologique. Nous rappelons l'aspect en imagerie du myélome multiple à localisation mandibulaire, en mettant l'accent sur les principaux diagnostics différentiels. Med Buccale Chir Buccale 2007; 13: 199-203.

mots clés: mandibule, myélome multiple, imagerie.

médecine

buccale chirurgie buccale

VOL. $13, \mathrm{~N}^{\circ} 4$ 2007

page 199

\section{SUMMARY}

We report a case of multiple myeloma in a 73-year-old male reveled by a mandibular tumefaction. The radiography of the mandible had shown an osseous destruction of the left mandibular ramus. CT scan had shown tumoral process of left mandible associated with radiolucent lesion of calvaria and destruction of the left pedicle of the third cervical vertebra suspecting the diagnosis of multiple myeloma. Positive diagnosis was confirmed by a biologic and radiological balance of myeloma. We discuss the imaging features of multiple myeloma with mandibular localization and review the differential diagnoses. Med Buccale Chir Buccale 2007; 13: 199-203.

key words: mandible, multiple myeloma, imaging.

\footnotetext{
1. Service d'Imagerie médicale, CHU Fattouma Bourguiba, Monastir, Tunisie

2. Service de Radiologie, Clinique hospitalo-universitaire de Médecine dentaire, Monastir, Tunisie

3. Service de Médecine et Chirurgie buccales, Clinique hospitalo-universitaire de Médecine dentaire, Monastir, Tunisie

Demande de tirés à part:
}

Habib Hamdi doc_hamdi@yahoo.fr 
Le myélome multiple est une hémopathie caractérisée par une prolifération monoclonale maligne de plasmocytes dans la moelle hématopoïétique [1, 2]. II s'agit d'une maladie très polymorphe, toutes les disciplines médicales pouvant être confrontées aux manifestations d'un myélome. Nous rapportons un cas de myélome multiple révélé par une tuméfaction mandibulaire. L'aspect en imagerie du myélome multiple à localisation mandibulaire et les principaux diagnostics différentiels sont discutés.

\section{OBSERVATION}

Un homme âgé de 73 ans, sans antécédents pathologiques particuliers, a consulté pour une tuméfaction mandibulaire gauche (Fig. 1). L'examen clinique a constaté une tuméfaction de la région de l'angle mandibulaire gauche, de

médecine

buccale

chirurgie

buccale

VOL. $13, \mathrm{~N}^{\circ} 4$ 2007

page 200 consistance molle et indolore. L'examen endobuccal a montré la présence d'une masse dépressible, comblant le fond du vestibule postéro-inférieur gauche. Le patient était édenté complet maxillaire et mandibulaire. L'orthopantomogramme a montré une lyse osseuse intéressant la région de l'angle et de la branche mandibulaire gauches, y compris le condyle et le processus coronoïde ; l'os mandibulaire résiduel a un aspect ostéoporotique diffus (Fig. 2). Une échographie en mode bidimensionnel et Doppler couleur de la tuméfaction a révélé un processus tissulaire hétérogène, mal limité et hypervascularisé (Fig. 3). L'examen tomodensitométrique du

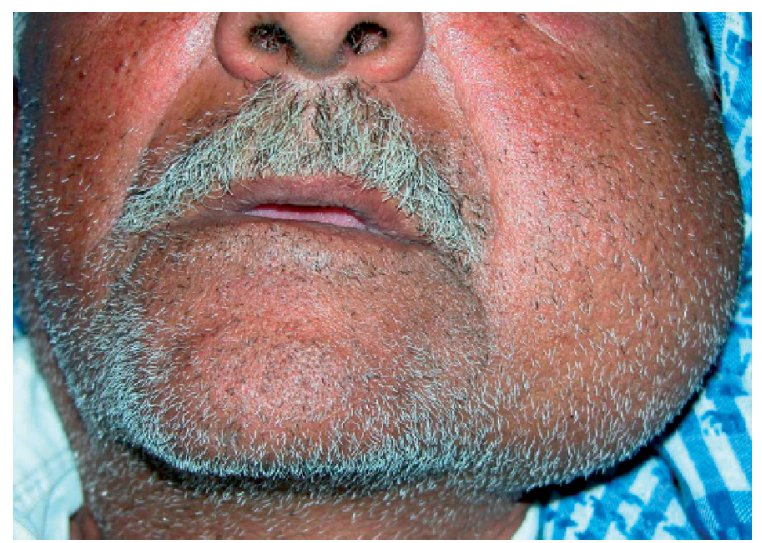

Figure 1 : Tuméfaction mandibulaire. Mandibular tumefaction.

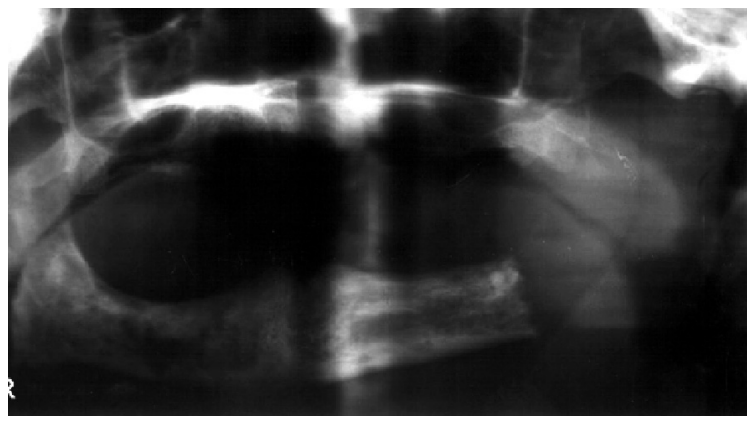

Figure 2 : Radiographie panoramique : ostéolyse totale du ramus mandibulaire gauche.

Panoramic radiograph: complete destruction of the left mandibular ramus.

massif facial et de la mandibule a montré une tumeur détruisant la partie postérieure du corps de la mandibule et la branche montante gauches, avec une extension aux espaces masticateur et submandibulaire, et un envahissement des muscles ptérygoïdiens médial et latéral (Fig. 4a-b).

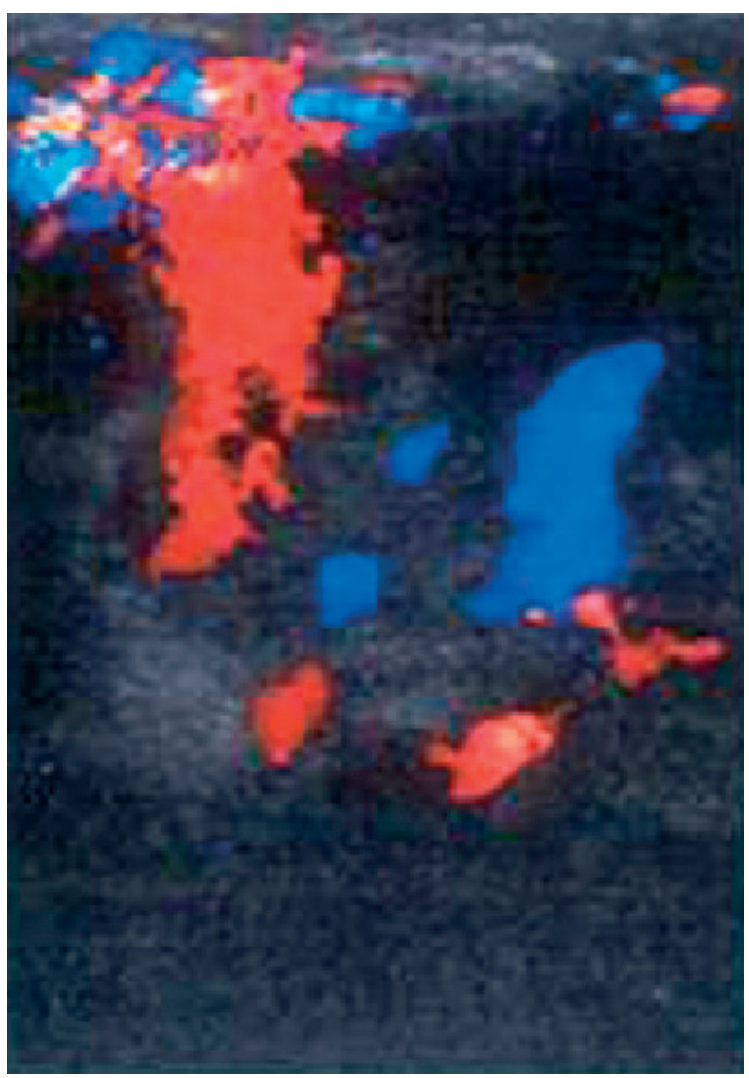

Figure 3 : Masse solide hypervascularisée en échographie Doppler.

Hypervascular solid mass in Doppler-ultrasound. 

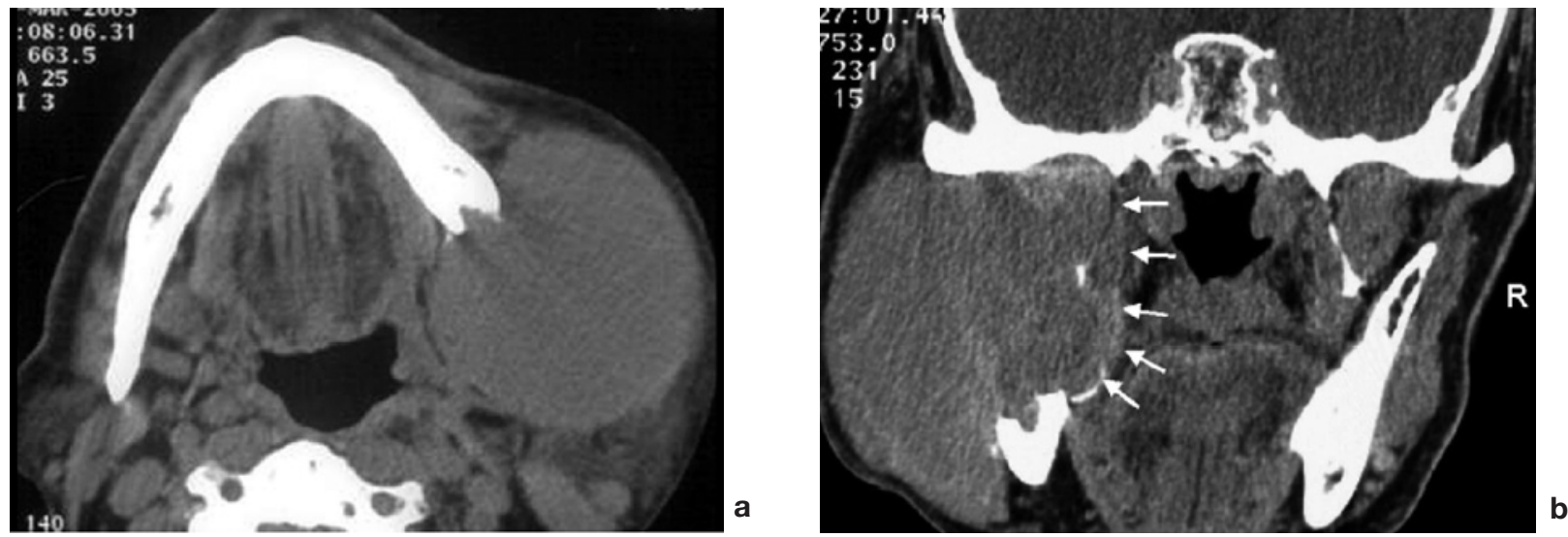

Figure 4 : Ostéolyse du ramus mandibulaire gauche avec envahissement des parties molles en TDM (flèches) :

a - coupe axiale,

$\mathrm{b}$ - coupe coronale.

A bony destructive lesion over left mandibular ramus with swelling of surrounding muscles in CT scan (arrows):

a - axial view,

b - coronal view.

L'étude en fenêtre osseuse et parenchymateuse des régions cervicales a objectivé une ostéolyse du pédicule gauche de la troisième vertèbre cervicale avec une extension dans le canal vertébral et dans les parties molles paravertébrales (Fig. 5). Une image lacunaire de la voûte crânienne droite a été découverte sur les coupes tomodensitométriques en fenêtre osseuse (Fig. 6). Devant ces

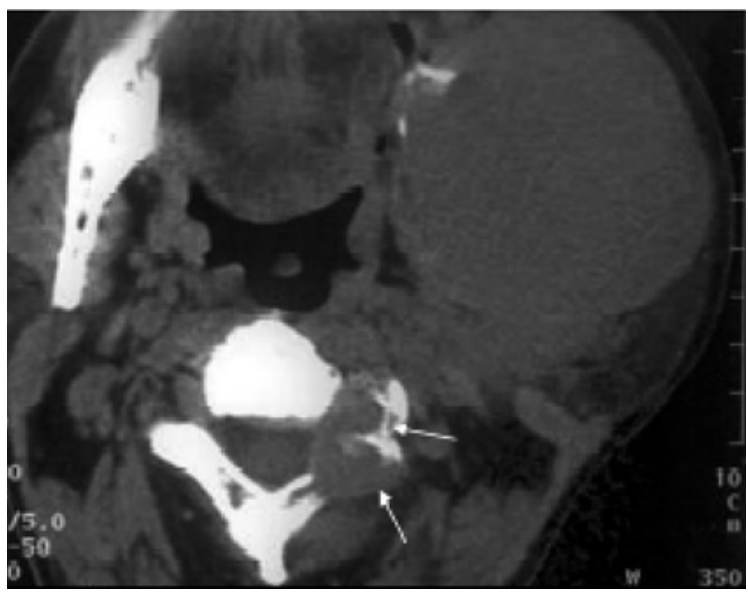

Figure 5 : Coupe axiale TDM passant par C3 montrant une ostéolyse du pédicule de la troisième vertèbre cervicale avec extension aux parties molles adjacentes (flèches).

Transverse CT image at level of C3 show destruction posterior arc of third cervical vertebra and extension into adjacent soft tissues (arrows). anomalies radiologiques, le diagnostic de myélome multiple a été suspecté. Ce diagnostic a été confirmé par le bilan biologique. Le patient a bénéficié d'une cure de chimiothérapie qui a entraîné une amélioration, une radiothérapie ciblée sur la tumeur mandibulaire a été préconisée mais le patient a été perdu de vue.

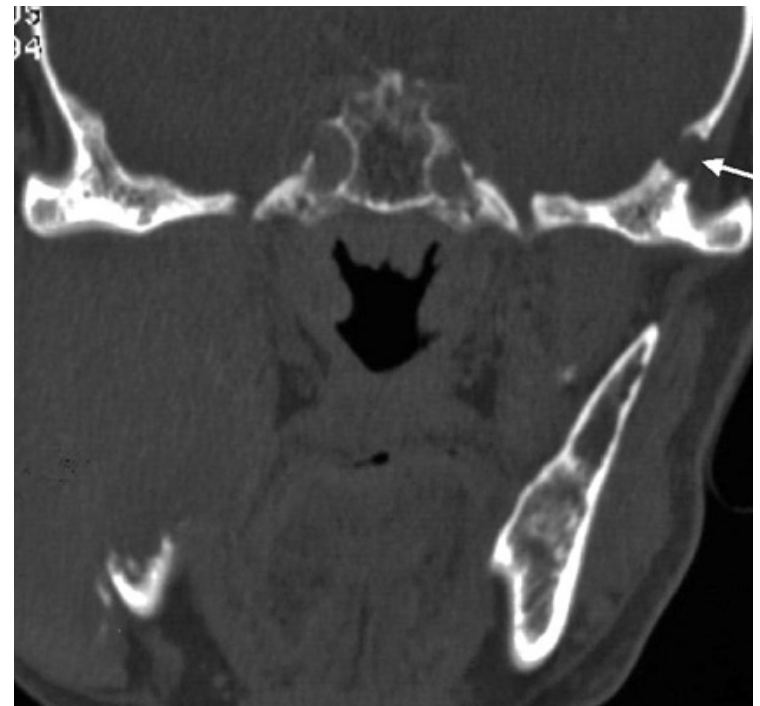

Figure 6 : TDM en coupe coronale : lacune à l'emporte pièce de la voûte crânienne du coté droit.

Coronal CT: a bony destructive lesion in the right dome of the skull. médecine

buccale

chirurgie

buccale

VOL. $13, N^{\circ} 4$ 2007

page 201 
médecine buccale chirurgie buccale

VOL. $13, \mathrm{~N}^{\circ} 4$ 2007

page 202

\section{COMMENTAIRES}

Le myélome multiple est constitué par une infiltration de la moelle par des plasmocytes néoplasiques qui sécrètent une immunoglobuline monoclonale et/ou une de ses chaînes constitutives. Il est responsable d'une ostéolyse diffuse. II est légèrement plus fréquent chez l'homme que chez la femme et l'âge moyen lors du diagnostic est de 65 ans [2]. L'atteinte du massif facial, et plus particulièrement de la mandibule, est rare. Lors d'une atteinte mandibulaire, la localisation postérieure est la plus fréquente en raison de la présence de moelle hématopoïétique dans cette région ${ }^{[3,4]}$. Les circonstances de découverte sont dominées par des manifestations osseuses ou des fractures pathologiques [1,3]. II est rare que le premier symptôme du myélome multiple soit constitué par une tumeur osseuse mandibulaire $[4,5]$. Les manifestations cliniques de la localisation mandibulaire sont variées : manifestations tumorales (voussure intrabuccale, tumeur rougeâtre...), perte de dents, hémorragie gingivale, troubles de la sensibilité [6]. L'orthopantomographie montre des images ostéolytiques, multiples, sans liseré d'ostéocondensation périphérique, siégeant sur un fond d'ostéoporose diffuse ; leur taille est variable, souvent petite, mais pouvant réaliser des ostéolyses extensives. Beaucoup plus rarement, on note la présence d'une soufflure ou d'une rupture de la corticale [7, 8]. Parfois, l'orthopantomographie révèle des lacunes à l'emporte pièce sans condensation périphérique ou réaction périostée, typiques de myélome [2, 9]. Dans l'observation que nous présentons, les radiographies standard ont montré une lyse de la région angulaire et de la totalité de la branche mandibulaire gauches. Ces radiographies restent insuffisantes pour affirmer la nature tumorale de la lésion et ne dispensent jamais d'un examen tomodensitométrique. Celuici est nécessaire et suffisant pour le diagnostic d'une tumeur mandibulaire ${ }^{[9]}$. L'image est celle d'une masse soufflant et détruisant les corticales, avec déplacement éventuel des dents, envahissement des parties molles adjacentes, et parfois fracture spontanée de la mandibule. Les lésions ostéocondensantes sont extrêmement rares au cours du myélome. Il s'agit souvent d'un liseré de condensation osseuse autour de la lésion [1]. Ces aspects radiologiques peuvent faire discuter un lymphome, des métastases, un ostéosarcome, voire un ostéochondrome ${ }^{[8,10]}$. Devant une tuméfaction mandibulaire et après un bilan radiologique standard, l'échographie permet de préciser les limites et la nature liquide ou solide de la lésion tumorale. Dans le myélome, la masse est d'échostructure tissulaire richement vascularisée et mal limitée. Une IRM peut être intéressante pour étudier l'envahissement des parties molles et, de plus, elle permet de mieux visualiser la moelle osseuse, point de départ de la maladie [1] ; elle trouve tout son intérêt dans le bilan initial comme dans le suivi de l'évolution après traitement. Dans l'observation que nous rapportons, le diagnostic de myélome multiple a été évoqué sur la TDM devant l'association lésion mandibulaire, images lacunaires de la voûte crânienne et lyse du pédicule gauche de la troisième vertèbre cervicale par une lésion qui intéresse les tissus du voisinage (parties molles paravertébrales et canal vertébral). Ce diagnostic a été confirmé par un myélogramme. Le traitement associe radiothérapie et chimiothérapie ${ }^{[6,9]}$. Le pronostic du myélome multiple est mauvais. La moyenne de survie avec traitement est de 48 mois malgré le traitement $[2,3]$. 


\section{RÉFÉRENCES}

1 - Elloumi M, Bellaaj H, Ayadi K, Zitoun I, Mseddi S, Frikha M, Kechaou M S. L'atteinte osseuse dans le myélome multiple : variétés et importance de la classification radiologique pour le pronostic. Tunis Med 2002 ; $80: 519-23$.

2 - Monje F, Gil-Diez JL, Campano FJ, Alonso del Hoyo JR. Mandibular lesion as the first evidence of multiple myeloma. J Craniomaxillofac Surg 1989 ; 17 : 315-7.

3 - Harousseau JL, Shaughnessy J, Richardson P. Multiple Myeloma. Hematology 2004 ; 1 : 237-56.

4 - Ho CL, Chen YC, Yiang YT, Kao WY, Chao TY, Mandibular mass as the presenting manifestation of lgm myeloma in a 22-year-old man. Ann Hematol 1999 ; 78 : 93-5.

5 - Lecouvet FE, Dechambre S, Malghem J, Ferrant A, Vande Berg BC, Maldaque B. Bone marrow transplantation in patients with multiple myeloma: prognostic significance of MR imaging. Am J Roentgen 2001 ; 176 : 91-6.
6 - Mozaffari E, Mupparapu M, Otis L. Undiagnosed multiple myeloma causing extensive dental bleeding: report of a case and review. Oral Surg Oral Med Oral Pathol Oral Radiol Endod 2002 ; 4 : 448-53.

7 - Bourjat P, Kahn JL, Braun JJ. Imagerie des plasmocytomes solitaires maxillo-mandibulaires. J Radiol 1999 ; 80 : 859-62.

8 - Chindia ML, Riyat MS, Nyong'o A. Multiple myeloma presenting as a painful mandibular swelling: a case report. Dent Update $2001 ; 28: 475$.

9 - Ustuner Z, Basaran M, Kiris T, Bilgic B, Sencer S, Sakar, Dizdar Y, Bavbek S, Onat H. Skull base plasmacytoma in a patient with light chain myeloma. Skull Base 2003 ; 13: 167-71.

10 - Lee SH, Huang JJ, Pan WL, Chan CP. Gingival mass as the primary manifestation of multiple myeloma: report of two cases. Oral Surg Oral Med Oral Pathol Oral Radiol Endod 1996 ; 82 : 75-9. \begin{tabular}{l}
$\begin{array}{l}\text { médecine } \\
\text { buccale } \\
\text { chirurgie } \\
\text { buccale }\end{array}$ \\
\hline VOL. $13, N^{\circ} 4$ \\
2007
\end{tabular}

page 203 